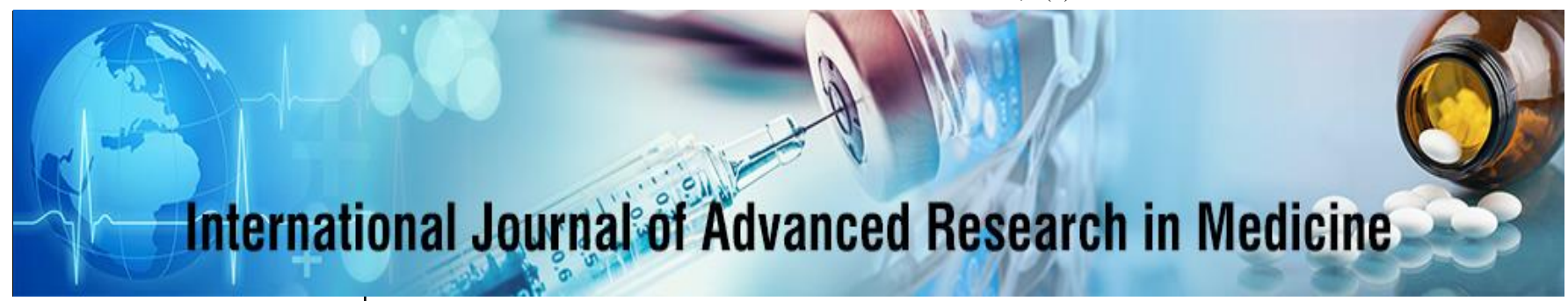

E-ISSN: 2706-9575

P-ISSN: 2706-9567

IJARM 2020; 2(2): 267-270

www.medicinepaper.net

Received: 25-05-2020

Accepted: 28-06-2020

Dr. Sunil Kumar Gulati MD Medicine, Metro Hospital and Cancer Research Center Jabalpur, Madhya Pradesh, India

\section{To determine the prevalence and severity of vitamin $D$ deficiency and its association with hyperglycaemia in type 2 DM}

\section{Dr. Sunil Kumar Gulati}

DOI: $\underline{\text { https://doi.org/10.22271/27069567.2020.v2.i2d.124 }}$

\begin{abstract}
Aims: The aim of the study to assess the prevalence and severity of vitamin D deficiency in type 2 DM as well as to record the effect of hyperglycaemia on serum vitamin D level.

Material and methods: A case-control study was conducted in the Department of General Medicine, Metro Hospital and Cancer Research Centre, Jabalpur, MP, India from December 2017 to November 2019. Total 240 participants were include in the study out of which 120 healthy people were include as case (Group A) and 120 type 2 diabetic patients as controls (Group B). Routine laboratory Investigations like CBC, FBS, RBS, PP2BS, HbA1C, blood urea, serum creatinine, lipid profile, urine albumin and Vitamin D3 levels were done by standard methods in central laboratory of Institute.

Results: Prevalence of low vitamin D level in healthy population was only $23.33 \%$ in my study, while prevalence was $85.83 \%$ in Diabetic group. Among diabetic patients having abnormal Vitamin D level, majority $(65 \%)$ were having insufficiency, only $20.83 \%$ were having over vitamin D deficiency in Diabetic patients. In patients with controlled diabetes as per $\mathrm{HbA1C}$ criteria, the prevalence of sufficient, Insufficient and Deficient Vitamin D was $18.51 \%, 61.11 \%$ and $16.67 \%$ respectively, where in patients with uncontrolled diabetes it was $10.60 \%, 68.18 \%$ and $24.24 \%$ respectively. More number of diabetic patients with uncontrolled status $(24.24 \%)$ was having overt vitamin D deficiency in comparison to controlled status (16.67\%). There is a significant association between the maintenance of euglycemia and severity of Vitamin D level in diabetic patients, as the p value is less than 0.05 . Hypertension was most common co-morbidity found in diabetic group (18.33\%) followed by Ischemic Heart Disease $(5.83 \%)$.

Conclusion: control of diabetic status is mandatory in order to prevent vitamin D deficiency.
\end{abstract}

Keywords: Serum vitamin D level, type 2 diabetes mellitus, vitamin D deficiency.

\section{Introduction}

Type 2 diabetes mellitus (T2DM) is a chronic metabolic disorder, the prevalence of which is increasing steadily all over the world. It has been estimated that, by 2030, the global population of diabetes would have been 562 million ${ }^{[1]}$. Although the number of people with T2DM is increasing in every country, its major contribution is from developing countries, where it is fast becoming an epidemic. Due to the increasing global burden of T2DM, the pathophysiology of this disease is being explored with renewed interest. Insulin resistance and $\beta$-cell failure are the core pathophysiologic defects of T2DM. It is primarily due to interplay between genetic and environmental factors. Incidence of T2DM varies from one geographical region to another due to differences in lifestyle and risk factors. Apart from conventional environmental risk factors like obesity, physical inactivity, intake of high calorie food and stress, the role of certain nutritional factors in pathogenesis of T2DM is an emerging concept at present. Accumulating evidence from several cross-sectional studies suggests that vitamin D has an important role in the homeostasis of blood glucose, and its deficiency may cause development of T2DM. Vitamin D, originally described merely as a vitamin, is indeed a misnomer as it is now well-established that its active form is a hormone which is not only involved in bone metabolism but also in a plethora of non-skeletal physiological processes. Several mechanisms have been proposed, indicating a positive effect of vitamin D on insulin secretion and sensitivity, which include its direct effect via activation of vitamin $\mathrm{D}$ receptor on pancreatic $\beta$-cells and insulin sensitive organs and indirect effect via regulation of calcium homeostasis ${ }^{[2,3]}$. In the largest epidemiological
Dr. Sunil Kumar Gulati MD Medicine, Metro Hospital and Cancer Research Center Jabalpur, Madhya Pradesh, India 
study from the NHANES population, a dose-dependent inverse relationship has been observed between vitamin D and type $2 \mathrm{DM}$ with the highest number of metabolic syndrome patients having the lowest quartiles of vitamin D ${ }^{[4]}$. The main source of vitamin D in humans is exposure to sunlight, natural diet and dietary supplements. Vitamin D from the skin and diet are metabolised in the liver to 25hydroxy vitamin $\mathrm{D}[25(\mathrm{OH}) \mathrm{D}]$ which is used to determine the patient's vitamin D status. Although there is no definite consensus about the normal level of vitamin $\mathrm{D}$, most experts define vitamin D deficiency as less than $20 \mathrm{ng} / \mathrm{ml}$. A level of $20-29 \mathrm{ng} / \mathrm{ml}$ is considered to indicate a relative insufficiency of vitamin D and a level of $30 \mathrm{ng} / \mathrm{ml}$ or greater can be considered as sufficient [5, 6] According to this definition, 1 billion people worldwide are suffering from vitamin D deficiency or insufficiency. Even in the sunniest countries including India, vitamin D deficiency is very common as most of the body surface is shielded from the sun. India is a country where both T2DM and hypovitaminosis D are prevalent. But relatively scarce data is available observing the correlation between the two. The aim of the study to assess the prevalence and severity of vitamin D deficiency in type $2 \mathrm{DM}$ as well as to record the effect of hyperglycaemia on serum vitamin D level.

\section{Material and Methods}

A case-control study was conducted in the Department of General Medicine, India from December 2017 to November 2019, after taking the approval of the protocol review committee and institutional ethics committee. After taking informed consent detailed history was taken from the patient or the relatives. The technique, risks, benefits, results and associated complications of the procedure were discussed with all patients.

\section{Methodology}

Total 240 participants were include in the study out of which 120 healthy people were include as case (Group A) and 120 type 2 diabetic patients as controls (Group B). Controls include age and sex matched healthy individuals. Patients with chronic kidney disease, patients taking calcium supplements or vitamin D supplements within last 3 months, patients suffering from any known chronic illness were excluded from this study. Routine laboratory Investigations like CBC, FBS, RBS, PP2BS, HbA1C, blood urea, serum creatinine, lipid profile, urine albumin and Vitamin D3 levels were done by standard methods in central laboratory of Institute. The value of serum vitamin D level was further divided in following category: sufficient $=30$ $100 \mathrm{ng} / \mathrm{ml}$, insufficient $=20-29 \mathrm{ng} / \mathrm{ml}$, deficiency $=$ less than $20 \mathrm{ng} / \mathrm{ml}$.

\section{Results}

In the study population, the mean age of group A (case) was $52.42 \pm 10.25$ years while that of group B (control) was $50.12 \pm 11.88$ years. Total 140 males and 100 females were enrolled in study. $65 \%$ of group A were male and 35 female. Group B (control) had 60\% males and 40 female. On evaluation of investigation profile of both group $A$ and group B, mean values of haematological parameters in form of haemoglobin, total count and platelet were within normal limit and comparable in both groups. Surprisingly mean value of renal function test parameters, blood urea and serum creatinine were within normal range for diabetic group also, though $10.5 \%$ of patients had abnormal serum creatinine value and it range from 2.22 to $4.2 \mathrm{mg} / \mathrm{dl}$. Mean value of all lipid profile component were in normal limit in both the group, but $35 \%$ of diabetic patients had dyslipidaemia and commonest dyslipidaemia was hypertriglyseridemia in 30\% patients. Frequency Distribution of Participants according to Severity of Vitamin D level noted. Prevalence of low vitamin D level in healthy population was only $23.33 \%$ in my study, while prevalence was $85.83 \%$ in Diabetic group. Among diabetic patients having abnormal Vitamin D level, majority (65\%) were having insufficiency, only $20.83 \%$ were having overt vitamin D deficiency in Diabetic patients (table 1.)

Table 1: Severity grading of vitamin d deficiency in cases and controls

\begin{tabular}{|c|c|c|c|c|}
\hline & Case $\mathbf{= 1 2 0}$ & $\mathbf{\%}$ & Control=120 & \% \\
\hline Deficiency & 25 & 20.83 & nil & \\
\hline Insufficient & 78 & 65 & 28 & 23.33 \\
\hline Sufficient & 17 & 14.17 & 92 & 76.67 \\
\hline
\end{tabular}

Table 2: The association of severity of vitamin D level with the category of diabetes control

\begin{tabular}{|c|c|c|c|c|}
\hline \multirow{2}{*}{ Diabetes control } & \multicolumn{3}{|c|}{ Vitamin D Level } & \multirow{2}{*}{ Chi Sq (p value) } \\
\cline { 2 - 4 } & Sufficient & Insufficient & Deficiency & \multirow{2}{*}{3.12} \\
\hline Controlled Diabetic $(\mathrm{N}=54)$ & $10(18.51 \%)$ & $33(61.11 \%)$ & $9(16.67 \%)$ & $(0.003)$ \\
\hline Uncontrolled Diabetic $(\mathrm{N}=66)$ & $7(10.60 \%)$ & $45(68.18 \%)$ & $16(24.24 \%)$ & \\
\hline
\end{tabular}

In patients with controlled diabetes as per $\mathrm{HbA} 1 \mathrm{C}$ criteria, the prevalence of sufficient, Insufficient and Deficient Vitamin D was $18.51 \%, 61.11 \%$ and $16.67 \%$ respectively, where in patients with uncontrolled diabetes it was $10.60 \%$, $68.18 \%$ and $24.24 \%$ respectively. More number of diabetic patients with uncontrolled status $(24.24 \%)$ was having overt vitamin $\mathrm{D}$ deficiency in comparison to controlled status $(16.67 \%)$. There is a significant association between the maintenance of euglycemia and severity of Vitamin D level in diabetic patients, as the $\mathrm{p}$ value is less than 0.05 (Table 2).

Pearson correlation test showed negative correlation between HbA1C level and mean vitamin D level in Diabetic group as $r=-0.266, p$ value $=<0.001$. It suggests as HbA1c level increase, the level of vitamin $\mathrm{D}$ decreases, so more severe the hyperglycaemia and poorer the control of diabetes status, there was more severe the vitamin D deficiency.

We also compare the mean value of vitamin D deficiency with the duration of Diabetes, but there was no significant relation between duration of diabetes and serum vitamin D deficiency $(\mathrm{P}$ value $>0.5)$.

Diabetic nephropathy was the most common micro vascular complication seen in type 2 diabetic patients. it was found that all three important microvascular complications: diabetic retinopathy, diabetic nephropathy and peripheral neuropathy did not have any significant correlation with serum Vitamin D level as p value is greater than 0.05 for all three parameters (Table 3). 
Table 3: Comparison of mean vitamin D level with duration and micro vascular complication of diabetes mellitus

\begin{tabular}{|c|c|c|c|c|}
\hline & Parameters & & Mean value of vitamin D (ng/dl) & p value \\
\hline & $0-5$ years & & $25.77 \pm 6.85$ & \\
\hline Duration of diabetes & $5-10$ years & & $27 \pm 5.85$ & 0.265 \\
\hline \multirow{7}{*}{ Micro-vascular complication } & $>10$ years & & $26.12 \pm 3.82$ & \\
\hline & Diabetic & Present & $24.11 \pm 5.02$ & \\
\hline & retinopathy & Absent & $26.31 \pm 5.22$ & 0.077 \\
\hline & Diabetic & Present & $27.11 \pm 5.45$ & \\
\hline & nephropathy & Absent & $25.41 \pm 6.35$ & 0.057 \\
\hline & Peripheral & Present & $24.24 \pm 4.69$ & \\
\hline & neuropathy & Absent & $27.25 \pm 5.88$ & 0.102 \\
\hline
\end{tabular}

Hypertension was most common co-morbidity found in diabetic group (18.33\%) followed by Ischemic Heart Disease $(5.83 \%)$. Serum vitamin D level was measured in all participants. 85.83 of diabetic population was having less than normal vitamin D level, while only $23.33 \%$ had less than normal vitamin $\mathrm{D}$ level in group $\mathrm{B}$ (control). Mean value of vitamin $\mathrm{D}$ in type 2 Diabetic patients was $26.33 \pm 6.94 \mathrm{ng} / \mathrm{dl}$ and mean value of vitamin $\mathrm{D}$ in healthy individuals was $35.67 \pm 4.88 \mathrm{ng} / \mathrm{dl}$.

Table 4: Subgroup analysis- mean vitamin D level in diabetes patients in relation with age, gender and associated co-morbidities

\begin{tabular}{|c|c|c|c|}
\hline \multicolumn{2}{|c|}{ Parameters (Number of patients) } & Mean value of vitamin D & p value \\
\hline Age group & Below40 (91) & $25.32 \pm 5.75$ & 0.289 \\
\hline & $>40(29)$ & $25.02 \pm 3.19$ & 0.027 \\
\hline Gender & Male (78) & $28.65 \pm 5.86$ & 0.033 \\
\hline HTN & Female (42) & $25.09 \pm 4.68$ & 0.125 \\
\hline Yes (22) & No (98) & $21.66 \pm 4.22$ & \\
\hline
\end{tabular}

\section{Discussion}

Vitamin D deficiency is a major health problem worldwide. The overall worldwide Vitamin D deficiency prevalence is around $15 \%$ according to study done by Pfotrnhauer KM et al. ${ }^{[7]}$ However the prevalence of vitamin D deficiency in India is around 50-90\% in normal healthy population. 8 In my study, prevalence of vitamin D deficiency is $23.33 \%$ in normal population which is similar to worldwide prevalence but very less in comparison to prevalence shown in Indian studies. This low prevalence in healthy population was contradictory to other Indian studies. Another on-going study on vitamin D level done in similar region had also showed $16 \%$ prevalence of vitamin D deficiency in healthy population. Vitamin D exerts its effect on calcium metabolism and hence affects skeletal system; however it also has extra skeletal effects like that on cardiovascular system, endocrine disorders and autoimmune disorder. Several reports have ascribed an active role to vitamin D in the functional regulation of the endocrine pancreas, particularly the beta-cells.

India is already declared as 'Capital of Diabetes'. Diabetes mellitus is accepted as major emerging epidemic in India, as India is having 41 million of diabetic patients currently and it will go up to 70 million by year 2025. As vitamin D has been showed to have effect on pathophysiology of diabetes and have very high prevalence of vitamin D deficiency, so we have taken up this study to see effect of both high prevalence diseases on each other.

Various studies done in different geographical region and cultural background have shown varied range of prevalence of vitamin $\mathrm{D}$ deficiency in diabetic group ranging from $67 \%-98.8 \%$ [8-11]. Our study along with Bashir et al. and Ifigenia-Kostoglou A et al. studies had shown higher prevalence of vitamin D deficiency in diabetes mellitus patients compared to healthy individuals, but two other studies had shown no difference of prevalence between diabetic and healthy population ${ }^{[8-11]}$. So, we have compared the mean value of serum vitamin $\mathrm{D}$ level in diabetic patients and in healthy population of various study. Various studies including our study had low mean level of vitamin D for diabetic patients in comparison to healthy population $[8,11$, 12].

In our study, mean vitamin D level is lower in patients with uncontrolled diabetes than patient with controlled diabetes ( $\mathrm{p}$ value $=0.003 \mathrm{Chi} \mathrm{Sq}$ test). Similar results was shown by Mukherjee B et al. Mean level of vitamin D is low in uncontrolled diabetic patients $(19.47 \pm 4.76)$ as compared to controlled diabetic patients $(23.63 \pm 3.71) .{ }^{12}$ Modi KD et al. found that vitamin $\mathrm{D}$ levels in patients with controlled diabetes was $22.4 \pm 18.6$ while in uncontrolled diabetic patients it was lower, $19.9 \pm 18.3$ which is statistically significant. ${ }^{13 \mathrm{~h}}$ Overall insufficiency is more common than deficiency state in diabetic patients regardless of diabetic control status, but severe vitamin D deficiency is more prevalent when patients were having uncontrolled diabetes than controlled diabetes (24.24\% and $16.67 \%$ respectively). On Pearson correlation, the study has demonstrated negative correlation between $\mathrm{HbA} 1 \mathrm{C}$ level and serum vitamin D level. It suggesting that as $\mathrm{HbA} 1 \mathrm{C}$ level increase, there is decrease in serum vitamin D level. Ifigenia-Kostoglou A et al. had also found that $25(\mathrm{OH})$ D3 levels were inversely associated with $\mathrm{HbA} 1 \mathrm{c}$ when the patient and control groups were analysed together $(\mathrm{p}=0.008, r 2=0.058$, linear regression analysis) ${ }^{[11]}$. Study by Mukherjee B et al. also indicates there is a definite negative correlation between Vitamin D levels and diabetes $(r=-0.94$ and -0.97$)$ and poorly controlled diabetics have further lower values of Vitamin D ${ }^{[12]}$. A study by Akshaykumar SV et al. showed a negative negligible co-relation between vitamin $\mathrm{D}$ levels and $\mathrm{HbA} 1 \mathrm{C}$, which was not statistically significant $(\mathrm{r}=$ 
$0.017, \mathrm{p}$ value 0.741$)^{[10]}$. The inverse relationship between vitamin D level and glycaemic control in this study support an active role of vitamin $\mathrm{D}$ in pathogenesis of type 2 diabetes mellitus.

Duration of diabetes and presence of micro-vascular complication do not have effect on serum vitamin $\mathrm{D}$ level. No effect of increasing age was observed on vitamin D status in diabetic patients and we could not able to find such association in other studies. Female diabetic patients were having lower vitamin $\mathrm{D}$ level compared to male counterparts; the reason might be less exposure to sun due to household activity. Hypertension was the most common comorbidity found in diabetic patients $(18.33 \%)$ in our study. Study by Shalini $\mathrm{P}$ et al. found that Vitamin D deficiency is more prevalent $(80.4 \%)$ in hypertensive patients than healthy $(67.7 \%)$ individuals ${ }^{[14]}$. Hypertensive diabetic patients had lower vitamin $\mathrm{D}$ level than nonhypertensive diabetic patients in my study, which is statistically significant as $\mathrm{p}$ value was 0.033 . Ischemic heart disease was another comorbidity found with diabetes, but there was no significant difference in mean Vitamin D level was recorded in diabetic patients with or without ischemic heart disease.

\section{Conclusion}

All patients of type 2 diabetes patients must be screened for vitamin $\mathrm{D}$ levels and those found to be having insufficiency or deficiency of vitamin D should be started on vitamin D supplements. Also strict control of diabetic status is mandatory in order to prevent vitamin D deficiency. We sincerely wish this study will be an important step in understanding association of Vitamin D level with type 2 diabetes mellitus and help in preventing vitamin D deficiency in diabetic patients.

\section{Reference}

1. Olokoba AB, Obateru OA, Olokoba LB. Type 2 Diabetes Mellitus: A Review of Current Trends. Oman Med J 2012;27(4):269-73.

2. Forouhi NG, Luan J, Cooper A et al. Baseline serum 25-hydroxy vitamin $\mathrm{D}$ is predictive of future glycaemic status and insulin resistance: the Medical Research Council Ely Prospective Study 1990 - 2000. Diabetes 2008;57:2619-25.

3. Chiu KC, Chu A, Go VL et al. Hypovitaminosis D is associated with insulin resistance and beta cell dysfunction. Am J Clin Nutr 2004;79:820-25.

4. Scragg R, Sowers M, Bell C. Serum 25-hydroxyvitamin $\mathrm{D}$, diabetes, and ethnicity in the Third National Health and Nutrition Examination Survey. Third National Health and Nutrition Examination Survey. Diab Care 2004;27(12):2813-8.

5. Holick MF, Siris ES, Binkley N et al. Prevalence of vitamin $\mathrm{D}$ inadequacy among postmenopausal North American women receiving osteoporosis therapy. J Clin Endocrinol Metab 2005;90:3215-24.

6. Lips P, Hosking D, Lippuner K et al. The prevalence of vitamin $\mathrm{D}$ inadequacy amongst women with osteoporosis: an international epidemiological investigation. J Intern Med 2006;260:245-54.

7. Pfotenhauer KM, Shubrook JH. Vitamin D deficiency, its role in health and disease, and current supplementation recommendations. J Am Osteopath Assoc 2017;117(5):301-5.
8. Laway BA, Kotwal SK, Shah ZA. Pattern of 25 hydroxy vitamin D status in North Indian people with newly detected type 2 diabetes: A prospective case control study. Indian J Endocrinol Meta 2014;18(5):726.

9. Alhumaidi M, Adnan AG, Dewish M. Vitamin D deficiency in patients with type-2 diabetes mellitus in southern region of Saudi Arabia. Maedica 2013;8(3):231.

10. Akshay Kumar SV, Nanda SK, Bharathy N, Ravichandran K, Dinakaran A, Ray L. Evaluation of vitamin D status and its correlation with glycated haemoglobin in type 2 diabetes mellitus. Biomedical Res 2017;28(1).

11. Kostoglou-Athanassiou I, Athanassiou P, Gkountouvas A, Kaldrymides P. Vitamin D and glycemic control in diabetes mellitus type 2. Therapeutic Adv Endocrinol Meta 2013;4(4):122-8.

12. Brijesh M, Saurav P. Prevalence of Vitamin D deficiency in type-2 Diabetes Mellitus patients and its correlation with glycaemic control. Int $\mathrm{J}$ Bioas 2014;3:3313-7.

13. Modi KD, Ahmed MI, Chandwani R, Kumar KH. Prevalence of vitamin D deficiency across the spectrum of glucose intolerance. $J$ Diabetes Meta Dis 2015;14(1):54.

14. Priya S, Singh A, Pradhan A, Himanshu D, Agarwal A, Mehrotra S. Association of Vitamin D and essential hypertension in a North Indian population cohort. Heart India 2017;5(1):7. 